\title{
THE INVERSE RESONANCE PROBLEM FOR PERTURBATIONS OF ALGEBRO-GEOMETRIC POTENTIALS
}

\author{
B. M. BROWN AND R. WEIKARD
}

\section{INTRODUCTION}

Algebro-geometric potentials $q$ of the one-dimensional Schrödinger operator $L=$ $-d^{2} / d x^{2}+q$ are a small but important class of potentials with a number of rather nice properties. Their name is due to the fact that there exists an a differential operator $P$ of odd order such that the pair $(P, L)$ satisfies the algebraic relation $P^{2}=R(L)$ for some polynomial $R$ of odd degree. Moreover, the commutator $[P, L]$ equals zero, so that, according to Lax's celebrated paper [13, $q$ is a stationary solution of some equation in the $\mathrm{KdV}$ hierarchy. However, the property which is most important for this paper is that the the solutions of the differential equation $-y^{\prime \prime}+q y=\lambda y$ are meromorphic functions of the independent variable (see Its and Matveev [11] and Segal and Wilson [15]). ${ }^{1}$ At least in some subclasses of algebro-geometric potentials this property allows to show that solutions have a certain algebraic structure (see Theorem 2). Asymptotic features of this structure survive when the potential is subjected to compactly supported perturbations. The paradigm of an algebro-geometric potential is the $g$-soliton $-g(g+1) / \cosh (x)^{2}$ (and the functions obtained from it under time evolution according to the $\mathrm{KdV}$ equation) but the set includes also rational, periodic, elliptic and other functions.

V. A. Marchenko [14 showed in 1955 that a real-valued potential $q$ on $[0, \infty)$ for which $(1+x) q(x)$ is integrable is uniquely determined from the scattering phase, the eigenvalues and their norming constants.

Neither the scattering phase (as a function on $\mathbb{R}$ ) nor the norming constants can be obtained directly from laboratory measurements and one might therefore ask what would constitute equivalent information. In the case of compactly supported potentials at least the answer is also (implicitly) given by Marchenko: Each piece of the required information can be obtained from the Jost function ${ }^{2}$ which, in this particular case, is an entire function. Thus it can be recovered from the location of its zeros (and its known asymptotic behavior along the positive imaginary line) with the aid of Hadamard's factorization theorem. From a physical point of view the squares of these zeros are (Dirichlet) eigenvalues or resonances (depending on whether the zero in question is in the upper or lower half plane). In other words, the location of eigenvalues and resonances determines a compactly supported realvalued potential.

Date: 23. May 2003.

${ }^{1}$ That meromorphic solutions of the differential equation are also a sufficient condition for $q$ to be algebro-geometric in at least the elliptic, simply periodic, and rational realm was shown in 9 and 17. See 10 for an overview of the subject.

${ }^{2}$ The solution $\psi(z, \cdot)$ of $-y^{\prime \prime}+q y=z^{2} y$ which asymptotically equals $\exp (i z x)$ is called the Jost solution of the problem; the function $\psi(\cdot, 0)$ is then called the Jost function. 
It is now important to realize that both eigenvalues and resonances appear as poles of the scattering amplitude. In contrast to eigenvalues the resonance poles are complex but they still produce a large bump in the scattering cross section for real energies if they are close enough to the real axis. Since the scattering cross section is measured in the laboratory the observation that eigenvalues and resonances determine the potential is very important from a practical point of view. To our knowledge this observation was first publicly made by Korotyaev 12 but Zworski [19] had realized (but not published) it earlier in the context of compactly supported even potentials on $\mathbb{R}$. Christiansen [7] is another recent contribution to this field.

In joint work with I. Knowles we have developed an approach to this kind of problems which is independent from Marchenko's (see [2]). Our approach makes no distinction whether $q$ is real or complex by reconstructing the Weyl-Titchmarsh $m$ function instead the scattering phase. The $m$-function may be defined for complex potentials (see Brown et. al. [3]) and determines the potential uniquely, just as in the case of real potentials (see 4 $)$ ). In this paper we will apply this new method to compactly supported perturbations of algebro-geometric potentials. Note that these potentials generally do not have an integrable first moment so that Marchenko's approach does not apply.

In Section 2 we present some basic facts about the Tichmarsh-Weyl $m$-function for complex-valued potentials. Section 3 states and proves a general theorem (Theorem 11) which identifies sufficient conditions under which the eigenvalues and resonances determine a potential. Algebro-geometric potentials are defined and discussed in Section 4. In particular, we show there that they provide examples for Theorem 11 Finally, in Section 5 we discuss compactly supported perturbations of algebro-geometric potentials. Theorem 4 is the main result of that section.

\section{Preliminaries}

Let $\Sigma$ be a fixed open sector of the complex plane whose vertex is at the origin. Then define $\mathcal{Q}_{\Sigma}$ to be the set of those complex-valued, locally integrable functions on $[0, \infty)$ for which there is an open half plane $\Lambda$ satisfying the following two requirements: ${ }^{3}$

(1) $\Lambda^{c} \cap \Sigma$ is bounded.

(2) The set $Q=\overline{\mathrm{co}}(\{q(x)+r: x, r \in[0, \infty)\})$ does not intersect $\Lambda$.

Conditions of this type have first been introduced by Brown et al. 3 . Given a function $q \in \mathcal{Q}_{\Sigma}$ we consider the differential expression $L=-d^{2} / d x^{2}+q$ on $[0, \infty)$. We will say that $q$ is of Class I, if at most one (up to constant multiples) solution of $L y=\lambda y$ is square integrable on $[0, \infty)$. Otherwise, if all solutions of $L y=\lambda y$ are square integrable on $[0, \infty)$, we will say that $q$ is of Class II. This classification is independent of the choice of $\lambda$. For real-valued potentials it coincides with the classical limit-point and limit-circle distinction. However, for complex-valued potentials it does not coincide with Sims's distinction (cf. [16]) between the limit-point and limit-circle cases. See [4] for a discussion of this issue.

\footnotetext{
${ }^{3}$ If $S$ is a subset of the complex plane we denote its complement by $S^{c}$ and its closed convex hull by $\overline{\mathrm{co}}(S)$.
} 
Now let $\theta(\lambda, \cdot)$ and $\phi(\lambda, \cdot)$ be linearly independent solutions of $L y=\lambda y$ satisfying the initial conditions ${ }^{4}$

$$
\begin{aligned}
\theta(\lambda, 0) & =1 & & \phi(\lambda, 0)=0 \\
\theta^{\prime}(\lambda, 0) & =0 & & \phi^{\prime}(\lambda, 0)=1 .
\end{aligned}
$$

It is shown in Brown et al. 3] (see also 4]) that for every $\lambda \in \Lambda$ there is at least one square integrable solution of $L y=\lambda y$ which is not a multiple of $\phi(\lambda, \cdot)$. Hence, if $q$ is of Class I and $\lambda \in \Lambda$, then there is precisely one square integrable solution $\chi(\lambda, \cdot)$ (up to constant multiples) and there is a unique number $m(\lambda)$ such that $\chi(\lambda, \cdot)=$ $\theta(\lambda, \cdot)+m(\lambda) \phi(\lambda, \cdot)$ is square integrable. This function $m: \Lambda \rightarrow \mathbb{C}: \lambda \mapsto m(\lambda)$ is the generalization of the Titchmarsh-Weyl $m$-function for a Dirichlet boundary condition at zero to the case of complex-valued potentials. Note that

$$
m(\lambda)=\frac{\chi^{\prime}(\lambda, 0)}{\chi(\lambda, 0)} .
$$

Just as in the selfadjoint case $m$ is an analytic function (see [3]). It may well be possible to extend it analytically to a larger domain than $\Lambda$. Sometimes $m$ may even be extended to the Riemann surface of $\lambda \mapsto \sqrt{\lambda}$. This is the case we are interested in and therefore we introduce the function

$$
M(z)=m\left(z^{2}\right)
$$

putting the branch cut on the positive real axis (so that $\operatorname{Im}(z)>0$ represents the so called physical $\lambda$-sheet).

\section{The MAIN THEOREM}

Definition 1. Given an odd polynomial $W \in \mathbb{C}[z]$ of degree $2 g+1$ define $\mathcal{C}_{W}$ to be the family of potentials $q \in \mathcal{Q}_{\Sigma}$ which are of Class I and for which there exist functions $\psi: \mathbb{C} \times[0, \infty) \rightarrow \mathbb{C}$ satisfying the following conditions:

(1) For every complex number $z$ the functions $\psi(z, \cdot)$ and $\psi(-z, \cdot)$ are nontrivial solutions of the differential equation $-y^{\prime \prime}+q y=z^{2} y$.

(2) The Wronskian of $\psi(z, \cdot)$ and $\psi(-z, \cdot)$ satisfies

$$
\psi(z, \cdot) \psi^{\prime}(-z, \cdot)-\psi(-z, \cdot) \psi^{\prime}(z, \cdot)=W(z)
$$

(3) $\psi(z, \cdot)$ is square integrable for all $z$ in some nonempty open subset of the upper halfplane $\mathbb{C}_{+}$.

(4) $\psi(\cdot, 0)$ and $\psi^{\prime}(\cdot, 0)$ are entire functions of finite growth order.

(5) There exists a ray such that $\psi(z, 0) / z^{g}$ tends to one as $z$ tends to infinity along the ray.

(6) There is an integer $p$ and a sequence of circles $t \mapsto r_{n} \exp (i t)$ such that $r_{n}$ tends to infinity and $\left|\left(\psi^{\prime} / \psi\right)\left(r_{n} \exp (i t), 0\right)\right| r_{n}^{-p-1}$ tends to zero uniformly for $t \in[0,2 \pi]$.

Theorem 1. Let $W \in \mathbb{C}[z]$ be an odd polynomial of degree $2 g+1$ and assume that $q$ is a potential in $\mathcal{C}_{W}$ with $\psi$ being the function from Definition 1 establishing that fact. If $W(z)=0$ implies $\psi(z, 0) \neq 0$ then the zeros of $\psi(\cdot, 0)$ and their multiplicities determine $q$ uniquely.

\footnotetext{
${ }^{4}$ Throughout the paper we will use the following notation for derivatives: If $f$ is a function of several variables we will use $\dot{f}$ and $f^{\prime}$ to denote the derivative of $f$ with respect to the first and last variable, respectively. If $f$ is a function of two variables $f^{(j, k)}$ denotes the function obtained by differentiating $j$ times with respect to the first variable and $k$ times with respect to the second.
} 
Proof. It is well-known that, in the self-adjoint case, the Titchmarsh-Weyl $m$ function determines the potential $q$ (see Bennewitz [1] for a rather concise proof). It was shown in 4 that this remains true even if $q$ is complex-valued at least as long as it is of Class I. Since, of course, $M$ determines $m$, we only have to show that the given information suffices to determine $M$.

It follows from condition (3) that

$$
M(z)=\frac{\psi^{\prime}(z, 0)}{\psi(z, 0)} .
$$

Condition (4) implies that $M$ is meromorphic and that its poles are the zeros of $\psi(\cdot, 0)$. We denote the poles of $M$ by the pairwise distinct numbers $z_{1}, z_{2}, \ldots$ and we use $n_{1}, n_{2}, \ldots$ for their respective multiplicities. The zeros are labelled such that $\left|z_{1}\right| \leq\left|z_{2}\right| \leq \ldots$

Let $h_{z}(\mu)=(z / \mu)^{p+1} /(z-\mu)$. Also define $\gamma_{n}(t)=r_{n} \exp (i t)$ for $t \in[0,2 \pi]$ and $B_{n}=\left\{z:|z|<r_{n}\right\}$. Then, by the residue theorem,

$$
\frac{1}{2 \pi i} \int_{\gamma_{n}} h_{z}(\mu) M(\mu) d \mu=-M(z)+\sum_{k=0}^{p} \frac{M^{(k)}(0)}{k !} z^{k}+\sum_{z_{j} \in B_{n}} \operatorname{res}_{z_{j}}\left(h_{z} M\right)
$$

if $0 \neq|z|<r_{n}$ and if $z$ is none of the poles of $M$. According to condition (6) the integral on the left tends to zero as $n$ tends to infinity proving firstly the convergence of the series and secondly that

$$
M(z)=\sum_{k=0}^{p} \frac{M^{(k)}(0)}{k !} z^{k}+\sum_{j=1}^{\infty} \operatorname{res}_{z_{j}}\left(h_{z} M\right) .
$$

Suppose we had already determined the infinite series on the right hand side of equation (11). We can then find the polynomial $\sum_{k=0}^{p} M^{(k)}(0) z^{k} / k$ ! from the asymptotic behavior of the $m$-function along some ray since $m\left(z^{2}\right)=i z+o(1)$ as $z$ tends to infinity along certain rays (see Theorem 6 of 2 ).

Thus the theorem is proved once we determine the residues of $h_{z} M$ at the poles of $M$. To do this let

Then

$$
f_{j}(\mu)=\frac{\left(\mu-z_{j}\right)^{n_{j}}}{\psi(\mu, 0)}
$$

$$
\begin{aligned}
\operatorname{res}_{z_{j}}\left(h_{z} M\right) & =\frac{1}{\left(n_{j}-1\right) !}\left(\psi^{\prime}(\cdot, 0) h_{z} f_{j}\right)^{\left(n_{j}-1\right)}\left(z_{j}\right) \\
& =\frac{1}{\left(n_{j}-1\right) !} \sum_{r=0}^{n_{j}-1}\left(\begin{array}{c}
n_{j}-1 \\
r
\end{array}\right) \psi^{(r, 1)}\left(z_{j}, 0\right)\left(h_{z} f_{j}\right)^{\left(n_{j}-1-r\right)}\left(z_{j}\right)
\end{aligned}
$$

and this quantity may be computed once we know the function $\psi(\cdot, 0)$ (and hence the functions $f_{j}$ ) and the numbers $\psi^{(r, 1)}\left(z_{j}, 0\right)$ for $r=0, \ldots, n_{j}-1$. We will now show that this information can be obtained from the given data.

Firstly, $\psi(\cdot, 0)$ is given through Hadamard's factorization theorem as

$$
\psi(z, 0)=z^{k} \exp (g(z)) \prod_{n=1}^{\infty} E_{\rho}\left(z / z_{n}\right)
$$

where $k$ and $\rho$ are integers, $g$ is a polynomial, and

$$
E_{\rho}(z)=(1-z) \exp \left(z+\frac{z^{2}}{2}+\ldots+\frac{z^{\rho}}{\rho}\right) .
$$


The number $\rho$ is to be chosen such that $\sum_{j=1}^{\infty} n_{j}\left|z_{j}\right|^{-\rho+1}$ is finite. This is always possible since otherwise $\psi(\cdot, 0)$ would not have finite growth order. The polynomial $g$ may be determined from the asymptotic behavior of $\psi(\cdot, 0)$, given in condition (5), and we have $k=0$ since $\psi(0,0) \neq 0$ (zero is a root of $W$ ).

Secondly, taking $r$ derivatives of the equation

$$
\psi(z, \cdot) \psi^{\prime}(-z, \cdot)-\psi(-z, \cdot) \psi^{\prime}(z, \cdot)=W(z)
$$

with respect to $z$ and evaluating them at $z_{j}$ gives that

$$
\psi^{(r, 1)}\left(z_{j}, 0\right) \psi\left(-z_{j}, 0\right)=-W^{(r)}\left(z_{j}\right)-\sum_{s=0}^{r-1} \frac{(-1)^{r-n} r !}{(r-n) ! n !} \psi^{(s, 1)}\left(z_{j}, 0\right) \psi^{(r-s, 0)}\left(-z_{j}, 0\right)
$$

as long as $r \leq n_{j}-1$ since $z_{j}$ is a zero of $\psi(\cdot, 0)=0$ of order $n_{j}$. We know that $\psi\left(-z_{j}, 0\right) \neq 0$ since $\psi\left(z_{j}, \cdot\right)$ and $\psi\left(-z_{j}, \cdot\right)$ are linearly independent. Hence the numbers $\psi^{(0,1)}\left(z_{j}, 0\right), \ldots, \psi^{\left(n_{j}-1,1\right)}\left(z_{j}, 0\right)$ may be computed recursively.

\section{Algebro-geometric potentials}

Let $L$ be the differential expression $L=-d^{2} / d x^{2}+q$. A meromorphic function $q$ is called algebro-geometric (or an algebro-geometric potential) if there exists an ordinary differential expression $P$ of odd order which commutes with $L$. The reason behind this choice of words is that, according to results of Burchnall and Chaundy [5], 6], the differential expressions $P$ and $L$ commute if and only if there exists a polynomial $\mathcal{Q}$ in two variables such that $\mathcal{Q}(P, L)=0$.

In this section we consider potentials which are either rational functions or else simply periodic meromorphic functions of period $p$ bounded at the ends of the period strip. We define

$$
\xi(x)= \begin{cases}x & \text { in the rational case } \\ \frac{p}{2 \pi i}\left(\mathrm{e}^{2 \pi i x / p}-1\right) & \text { in the periodic case. }\end{cases}
$$

and

or explicitly

$$
\mathcal{P}(x)=\frac{\xi^{\prime}(x)}{\xi(x)^{2}}
$$

$$
\mathcal{P}(x)= \begin{cases}1 / x^{2} & \text { in the rational case, } \\ \left(\frac{2 \pi i}{p}\right)^{2} \mathrm{e}^{2 \pi i x / p} /\left(\mathrm{e}^{2 \pi i x / p}-1\right)^{2} & \text { in the periodic case. }\end{cases}
$$

Note that, as $p$ tends to infinity, $\xi(x)$ tends to $x$. It will be convenient to refer to the rational case as the case where $p$ is infinite.

Remarks:

(1) $\mathcal{P}(x)$ tends to zero if $x$ tends to the ends of the period strip (defining the whole complex plane as the period strip if $p$ is infinite). Moreover, the principal part of $\mathcal{P}$ at zero equals $1 / x^{2}$.

(2) It was shown in [17 that the potential $q_{0}$ is algebro-geometric if and only if all solutions of the equation $-y^{\prime \prime}+q_{0} y=\lambda y$ are meromorphic for all complex numbers $\lambda$ provided that $q_{0}$ is either rational or else simply periodic, meromorphic, and bounded at the ends of the period strip. This provides a simply criterion by which one may determine whether a potential is algebro-geometric. 
Theorem 2. Suppose that $q_{0}$ is a rational function bounded at infinity or a simply periodic meromorphic function bounded at the ends of the period strip and that $q_{0}$ is algebro-geometric. Then the following statements hold:

(1) $q_{0}(x)=\lambda_{0}+\sum_{j=1}^{m} s_{j}\left(s_{j}+1\right) \mathcal{P}\left(x-x_{j}\right)$ for a suitable choice of the parameters $\lambda_{0}, m, s_{1}, \ldots, s_{m}$ (we let $m=0$ if $q_{0}$ is constant) and suitable pairwise distinct (modulo the period in the periodic case) points $x_{1}, \ldots, x_{m}$.

(2) There is a nonnegative integer $g$ and there are rational functions $r_{0}, \ldots, r_{g-1}$ such that $\psi_{0}(z, x)=\left(z^{g}+r_{g-1}(\xi(x)) z^{g-1}+\ldots+r_{0}(\xi(x))\right) \exp (i z x)$ is a solution of the equation $-y^{\prime \prime}+\left(q_{0}-\lambda_{0}\right) y=z^{2} y$.

(3) The Wronskian $W$ of $\psi_{0}(z, \cdot)$ and $\psi_{0}(-z, \cdot)$ is an odd polynomial in $\mathbb{C}[z]$ of degree $2 g+1$. In the rational case its only zero is $z=0$, i.e., $W(z)=$ $-2(i z)^{2 g+1}$. In the periodic case all zeros of $W$ are simple.

Proof. Statement (1) was proved in [17. It was proved in [18] that $-y^{\prime \prime}+q_{0} y=\lambda y$ has at least one solution $\psi_{0}(z, \cdot)$ of the form given. It is then a straightforward calculation to show that $\psi_{0}(-z, \cdot)$ also yields a solution. This gives statement (2). Statement (3) was proved in 8 .

These results enable us to show that $q_{0}$ is in $\mathcal{C}_{W}$ where $W$ is the Wronskian just introduced: Theorem 2 shows the validity of condition (1). Condition (2) is satisfied by definition. If $q_{0}$ is rational then $\psi_{0}(z, \cdot)$ is square integrable for any $z$ in the upper half plane. In the periodic case $\mathrm{e}^{i z x}$ decays faster than any power of $\xi(x)$ provided that $\operatorname{Im}(z)$ is sufficiently large. Hence $\psi_{0}(z, \cdot)$ is square integrable for any $z$ with sufficiently large imaginary part. This proves that condition (3) holds. Obviously $\psi_{0}(\cdot, 0)$ as well as $\psi_{0}^{\prime}(\cdot, 0)$ are polynomials and hence entire functions of growth order zero. Condition (5) holds for any ray in the complex plane. Since

$$
M(z)=\frac{\psi^{\prime}(z, 0)}{\psi(z, 0)}=i z+O\left(z^{-1}\right)
$$

condition (6) is satisfied with $p=1$. The circles may eventually be chosen arbitrarily as $M$ has only finitely many poles.

\section{Compactly supported perturbations of BAse potentials}

5.1. Transformation operators. Throughout this section we require the following hypothesis to be satisfied.

Hypothesis 1. $q$ and $q_{0}$ are locally integrable functions on $[0, \infty)$ and there exists a positive number $R$ such that the support of $q-q_{0}$ is contained in $[0, R]$. Moreover, the following estimate holds:

$$
M=\sup \left\{\int_{0}^{(t-x) / 2}\left|q(\alpha-\beta)-q_{0}(\alpha+\beta)\right| d \beta: \frac{t-x}{2} \leq \alpha \leq R, 0 \leq x \leq t\right\}<\infty .
$$

Let $\Omega=\left\{(t, x) \in \mathbb{R}^{2}: 0 \leq x \leq t\right\}$ and $\Omega_{0}=\left\{(t, x) \in \mathbb{R}^{2}: 0 \leq x \leq t \leq 2 R-x\right\}$. For $(t, x) \in \Omega$ define

$$
K_{0}(t, x)=\frac{1}{2} \int_{(t+x) / 2}^{\infty}\left(q(s)-q_{0}(s)\right) d s
$$

and, for $n \in \mathbb{N}$,

$$
K_{n}(t, x)=\int_{(t+x) / 2}^{\infty} \int_{0}^{(t-x) / 2}\left(q(\alpha-\beta)-q_{0}(\alpha+\beta)\right) K_{n-1}(\alpha+\beta, \alpha-\beta) d \beta d \alpha .
$$


Lemma 1. If $\left(q, q_{0}\right)$ satisfies Hypothesis 1 then, for every nonnegative integer $n$,

$$
\left|K_{n}(t, x)\right| \leq \frac{1}{2} \frac{M^{n}}{n !}\left(R-\frac{t+x}{2}\right)_{+}^{n} \int_{(t+x) / 2}^{R}\left|q(s)-q_{0}(s)\right| d s .
$$

In particular, $K_{n}(t, x)=0$ if $t+x \geq 2 R$.

Proof. The lemma will proved by induction on $n$. The statement is true for $n=0$. Assume now that it holds for $n-1$.

If $t+x \geq 2 R$ then $K_{n-1}(\alpha-\beta, \alpha+\beta)=0$ for all $(\alpha, \beta)$ in the domain of integration and hence $K_{n}(t, x)=0$.

If $t+x \leq 2 R$ we obtain

$$
\begin{aligned}
\left|K_{n}(t, x)\right| & \leq \int_{(t+x) / 2}^{R} \int_{0}^{(t-x) / 2}\left|q(\alpha-\beta)-q_{0}(\alpha+\beta)\right|\left|K_{n-1}(\alpha+\beta, \alpha-\beta)\right| d \beta d \alpha \\
& \leq \frac{1}{2} \frac{M^{n-1}}{(n-1) !} \int_{(t+x) / 2}^{R}(R-\alpha)^{n-1} \int_{\alpha}^{R}\left|q(s)-q_{0}(s)\right| d s M d \alpha \\
& \leq \frac{1}{2} \frac{M^{n}}{(n-1) !} \int_{(t+x) / 2}^{R}\left|q(s)-q_{0}(s)\right| d s \int_{(t+x) / 2}^{R}(R-\alpha)^{n-1} d \alpha .
\end{aligned}
$$

Because of this lemma the function

$$
K(t, x)=\sum_{n=0}^{\infty} K_{n}(t, x)
$$

is well defined for $0 \leq x \leq t$ and satisfies the integral equation

$$
\begin{aligned}
K(t, x)= & \frac{1}{2} \int_{(t+x) / 2}^{R}\left(q(s)-q_{0}(s)\right) d s \\
& +\int_{(t+x) / 2}^{R} \int_{0}^{t-x}\left(q(\alpha-\beta)-q_{0}(\alpha+\beta)\right) K(\alpha+\beta, \alpha-\beta) d \beta d \alpha .
\end{aligned}
$$

We also define

$$
H(t, x)=\int_{(t+x) / 2}^{R} \int_{0}^{t-x} h(\alpha, \beta) d \beta d \alpha=\sum_{n=1}^{\infty} K_{n}(t, x)
$$

where

$$
h(\alpha, \beta)=\left(q(\alpha-\beta)-q_{0}(\alpha+\beta)\right) K(\alpha+\beta, \alpha-\beta)
$$

so that

$$
K(t, x)=K_{0}(t, x)+H(t, x) .
$$

Lemma 2. If $\left(q, q_{0}\right)$ satisfies Hypothesis 1 the following statements hold:

(i) $K$ is continuous on $\Omega$ and $H$ is continuously differentiable on $\Omega$.

(ii) For $x \in[0, R]$ the functions $K(\cdot, x), H^{(1,0)}(\cdot, x)$, and $H^{(0,1)}(\cdot, x)$ are uniformly absolutely continuous on $[x, \infty)$. For $t \in(0, \infty)$ the functions $K(t, \cdot), H^{(1,0)}(t, \cdot)$, and $H^{(0,1)}(t, \cdot)$ are uniformly absolutely continuous on $[0, t]$. Moreover,

$$
H^{(2,0)}(t, x)-H^{(0,2)}(t, x)=-\left(q(x)-q_{0}(t)\right) K(x, t) .
$$


Proof. If $f$ is an absolutely continuous function both $x \mapsto f(x+t)$ and $t \mapsto f(x+$ $t)$ are uniformly absolutely continuous. Hence this is the case for $K_{0}(\cdot, x)$ and $K_{0}(t, \cdot)$. Next one proves by induction that $K_{n}(\cdot, x)$ and $K_{n}(t, \cdot)$ are continuously differentiable and that their derivatives converge uniformly.

Next note that

$$
H^{(1,0)}(t, x)=-\frac{1}{2} \int_{0}^{(t-x) / 2} h((t+x) / 2, \beta) d \beta+\frac{1}{2} \int_{(t+x) / 2}^{R} h(\alpha,(t-x) / 2) d \alpha
$$

and

$$
H^{(0,1)}(t, x)=-\frac{1}{2} \int_{0}^{(t-x) / 2} h((t+x) / 2, \beta) d \beta-\frac{1}{2} \int_{(t+x) / 2}^{R} h(\alpha,(t-x) / 2) d \alpha .
$$

Uniform absolute continuity of these functions is then shown directly using the uniform absolute continuity of $K$. The last claim follows by direct computation.

Theorem 3. Assume that Hypothesis 1 is satisfied. If $y_{0}$ is in the maximal domain of $-d^{2} / d x^{2}+q_{0}$ then $y$, given by

$$
y(x)=y_{0}(x)+\int_{x}^{2 R} K(t, x) y_{0}(t) d t
$$

is in the maximal domain of $-d^{2} / d x^{2}+q$. Moreover, if $-y_{0}^{\prime \prime}+q_{0} y_{0}=z^{2} y_{0}$, then $-y^{\prime \prime}+q y=z^{2} y$.

Proof. Since $y$ is eventually equal to $y_{0}$ and continuously differentiable we only have to show that $y^{\prime}$ is locally absolutely continuous and that $-y^{\prime \prime}+q y$ is locally square integrable.

One computes

$$
y^{\prime}(x)=y_{0}^{\prime}(x)-K(x, x) y_{0}(x)+\int_{x}^{2 R} K_{0}^{(0,1)}(t, x) y_{0}(t) d t+\int_{x}^{2 R} H^{(0,1)}(t, x) y_{0}(t) d t .
$$

The preceding lemma shows that the last term on the right is locally absolutely continuous. The substitution $t+x=2 u$ shows that the third term on the right is also locally absolutely continuous and that its derivative is

$$
\frac{1}{2}\left(q-q_{0}\right)(x) y_{0}(x)-\int_{x}^{2 R} K_{0}^{(0,1)}(t, x) y_{0}^{\prime}(t) d t
$$

Therefore

$$
\begin{aligned}
y^{\prime \prime}(x)= & y_{0}^{\prime \prime}(x)-K(x, x) y_{0}^{\prime}(x)-\left(K^{(1,0)}+K^{(0,1)}\right)(x, x) y_{0}(x)+\frac{1}{2}\left(q-q_{0}\right)(x) y_{0}(x) \\
& -\int_{x}^{2 R} K_{0}^{(0,1)}(t, x) y_{0}^{\prime}(t) d t-H^{(0,1)}(x, x) y_{0}(x)+\int_{x}^{2 R} H^{(0,2)}(t, x) y_{0}(t) d t .
\end{aligned}
$$

Two integrations by parts show that

$$
\begin{aligned}
\int_{x}^{2 R} K_{0}^{(0,1)}(t, x) y_{0}^{\prime \prime}(t) d t= & -K(x, x) y_{0}^{\prime}(x)+H^{(1,0)}(x, x) y_{0}(x) \\
& -\int_{x}^{2 R} K_{0}^{(1,0)}(t, x) y_{0}^{\prime}(t) d t+\int_{x}^{2 R} H^{(2,0)}(t, x) y_{0}(t) d t .
\end{aligned}
$$

Using that $K_{0}^{(1,0)}=K_{0}^{(0,1)}$ and equation (3) yields

$$
-y^{\prime \prime}(x)+q(x) y(x)=-y_{0}^{\prime \prime}(x)+q_{0}(x) y_{0}(x)+\int_{x}^{2 R} K(t, x)\left(-y_{0}^{\prime \prime}(t)+q_{0}(t) y(t)\right) d t
$$


which completes the proof of the theorem.

Hypothesis 2. For some nonnegative integer $n$ the functions $q$ and $q_{0}$ have the properties:

(i) $q \in A C^{n}([0, R])$ and $q_{0} \in A C^{n}([0,2 R])$.

(ii) $q^{(j)}(R)=q_{0}^{(j)}(R)$ for $j=0, \ldots, n-1$ but $q^{(n)}(R) \neq q_{0}^{(n)}(R)$.

Lemma 3. Suppose that the hypotheses 1 and 0 are satisfied. Then the following statements hold:

(i) $K \in C^{n}(\Omega)$ and $K \in C^{n+1}\left(\Omega_{0}\right)$.

(ii) $K^{(n+1,0)}(2 R, 0)=-\left(q-q_{0}\right)^{(n)}(R) / 2^{n+2} \neq 0$.

(iii) If $(t, x) \in \Omega_{0}$ and $k+\ell=n+1$ then $K^{(k, \ell)}(\cdot, x) \in A C([x, 2 R-x])$ and $K^{(k, \ell)}(t, \cdot) \in A C([0, t])$.

Proof. Since $q$ and $q_{0}$ are $n$ times continuously differentiable every derivative of order $r$ of $\int_{(t+x) / 2}^{R}\left(q-q_{0}\right)(s) d s$ is given by

$$
-\frac{1}{2^{r}}\left(q-q_{0}\right)^{(r-1)}((t+x) / 2)
$$

provided that $1 \leq r \leq n+1$ and that $(t, x) \in \Omega_{0}$. We therefore have to investigate only the function $H$. Let $\Omega_{0}^{\prime}=\left\{(\alpha, \beta) \in \mathbb{R}^{2}: 0 \leq \beta \leq \alpha \leq R\right\}$. Induction shows that $h \in C^{r-1}\left(\Omega_{0}^{\prime}\right)$ implies that there are numbers $a_{k, \ell, j}, b_{k, \ell}$, and $c_{k, \ell}$ such that

$$
\begin{aligned}
H^{(k, \ell)}(t, x)= & \sum_{j=0}^{k+\ell-2} a_{k, \ell, j} h^{(j, k+\ell-2-j)}\left(\frac{t+x}{2}, \frac{t-x}{2}\right) \\
& +b_{k, \ell} \int_{0}^{(t-x) / 2} h^{(k+\ell-1,0)}\left(\frac{t+x}{2}, \beta\right) d \beta \\
& +c_{k, \ell} \int_{(t+x) / 2}^{R} h^{(0, k+\ell-1)}\left(\alpha, \frac{t-x}{2}\right) d \alpha
\end{aligned}
$$

provided that $1 \leq k+\ell=r$. On the other hand

$$
h^{(k, \ell)}(\alpha, \beta)=\sum_{\substack{\nu, \mu \geq 0 \\ \nu+\mu \leq k+\ell}} g_{k, \ell, \nu, \mu} K^{(\nu, \mu)}(\alpha+\beta, \alpha-\beta)
$$

where

$$
g_{k, \ell, \nu, \mu}=\tilde{a}_{k, \ell, \nu, \mu} q^{(k+\ell-\nu-\mu)}(\alpha-\beta)+\tilde{b}_{k, \ell, \nu, \mu} q_{0}^{(k+\ell-\nu-\mu)}(\alpha+\beta)
$$

for certain numbers $\tilde{a}_{k, \ell, \nu, \mu}$ and $\tilde{b}_{k, \ell, \nu, \mu}$. Hence $g \in C^{r}\left(\Omega_{0}^{\prime}\right)$ as long as $k+l=r \leq n$. This shows that $K \in C^{n+1}\left(\Omega_{0}\right)$. We know already that $K$ is identically zero outside $\Omega_{0}$. To show that $K \in C^{n}(\Omega)$ we need $K^{(k, \ell)}(t, x)=0$ when $t+x=2 R$ and $k+\ell \leq n$. But

$$
K^{(k, \ell)}(t, x)=-\frac{1}{2^{k+\ell+1}}\left(q-q_{0}\right)^{(k+\ell-1)}((t+x) / 2)+H^{(k, \ell)}(t, x) .
$$

If $t+x=2 R$ then the first term on the right is zero if $k+\ell \leq n$. Using (4) and (5), the second term is expressed as a sum of terms involving expressions of the form $K^{(r, s)}(R+\beta, R-\beta)$ where $r+s \leq k+\ell-1$ so that one can show inductively that $H^{(k, \ell)}(t, x)=0$ when $t+x=2 R$ even if $k+\ell=n+1$. This completes the proof of (i) and (ii). 
To prove (iii) we have to investigate (6) again but for variable $t$ (or $x$ ) and $k+\ell=n+1$. The first term is absolutely continuous with respect to either variable in the stated intervals by assumption. The second term is treated in the same way as $H^{(1,0)}$ or $H^{(0,1)}$ were treated in Lemma 2

5.2. The location of the resonances. In this section we want to study the asymptotic location of resonances for compactly supported perturbations of algebrogeometric potentials. Therefore we assume henceforth the validity of the following hypothesis.

Hypothesis 3. $q_{0}$ is an algebro-geometric potential of the form

$$
q_{0}(x)=\lambda_{0}+\sum_{j=1}^{m} s_{j}\left(s_{j}+1\right) \mathcal{P}\left(x-x_{j}\right)
$$

where $\mathcal{P}$ is the function defined in (2) and where $\left\{x_{j}+n p: n \in \mathbb{Z}, j=1, \ldots, m\right\} \cap$ $[0, \infty)=\emptyset$ (for infinite $p$ this just means $\left\{x_{j}: j=1, \ldots, m\right\} \cap[0, \infty)=\emptyset$ ).

$q$ is a perturbation of $q_{0}$ such that $\left(q, q_{0}\right)$ satisfies hypotheses 1 and 2

Under this hypothesis $q_{0}$ is real analytic on $[0, \infty)$. Moreover, the functions $r_{j}$ introduced in Theorem 2 satisfy the estimate

$$
\left|r_{j}(\xi(x))\right| \leq \rho
$$

for some number $\rho$ independent of $j \in\{0, \ldots, g-1\}$ and $x \in[0,2 R]$.

Recall that

$$
\psi_{0}(z, x)=\left(z^{g}+r_{g-1}(\xi(x)) z^{g-1}+\ldots+r_{0}(\xi(x))\right) \exp (i z x)
$$

and define

$$
\varphi(z, x)=\int_{x}^{2 R} K(t, x) \psi_{0}(z, t) d t=\sum_{j=0}^{g} z^{j} \int_{x}^{2 R} K(t, x) r_{j}(\xi(t)) \exp (i z t) d t
$$

and

$$
\psi(z, x)=\psi_{0}(z, x)+\varphi(z, x) .
$$

Lemma 4. Suppose $\left(q, q_{0}\right)$ satisfies Hypothesis 3 . Then the following statements hold:

(1) Let $\operatorname{Im}(z)$ be fixed. Then $z^{-g} \varphi(z, x)$ tends to zero as $\operatorname{Re}(z)$ tends to $\pm \infty$.

(2) $z^{-g} \varphi(z, x)$ tends to zero uniformly in $\operatorname{Re}(z)$ as $\operatorname{Im}(z) \geq 0$ tends to $\infty$.

Proof. By the Riemann-Lebesgue lemma $\int_{0}^{2 R} K(t, 0) r_{j}(\xi(t)) \exp (i z t) d t$ tends to zero as $\operatorname{Re}(z)$ tends to infinity. This proves Statement (11). To prove (2) note that

$$
\left|z^{-g} \varphi(z, x)\right| \leq \sum_{j=0}^{g}|z|^{j-g} \int_{0}^{2 R}\left|K(t, 0)\left\|r_{j}(\xi(t)) \mid \mathrm{e}^{-\operatorname{Im}(z) t} d t \leq \frac{\rho \mathrm{e}^{M R}}{2 \operatorname{Im}(z)}\right\| q-q_{0} \|_{1}\right.
$$

if $|z| \geq 1$.

Lemma 5. Let $\nu$ be a positive number and $c_{1}$ a non-zero complex number. Suppose that

$$
\varphi(z, 0)=\int_{0}^{2 R} K(t, 0) \psi_{0}(z, t) d t=z^{g}\left(c_{1} z^{-\nu} \mathrm{e}^{2 i z R}\left(1+f_{1}(z)\right)+f_{2}(z)\right)
$$


where $\left|f_{1}(z)\right| \leq 1 / 12$ and $\left|f_{2}(z)\right| \leq 1 / 6$ for all sufficiently large $z$ in the closed lower half plane $\operatorname{Im}(z) \leq 0$. Then there is a number $\tau$ such that $|\psi(z, 0)| \geq|z|^{g} / 3$ for all $z$ on the semicircles given by $|z|=(2 n \pi+\tau) /(2 R)$ and $\operatorname{Im}(z) \leq 0$ and sufficiently large integers $n$.

Proof. Recall that $\psi(z, 0)=\psi_{0}(z, 0)+\varphi(z, 0)$ and that $\left|z^{-g} \psi_{0}(z, 0)-1\right| \leq 1 / 12$ when $|z|$ is sufficiently large.

We write $x=\operatorname{Re}(z), y=\operatorname{Im}(z)$, and $c_{1}=\mathrm{e}^{\sigma+i \kappa}$ where $\sigma, \kappa \in \mathbb{R}$. To prove the lemma we distinguish three cases.

First case: $|\operatorname{Im}(z)| \leq(\nu \log (n \pi / R)-\sigma-2) /(2 R)$ :

In this case $\varphi(z, 0)$ is negligible as compared to $z^{g}$ since

$$
\left|c_{1} z^{-\nu} \mathrm{e}^{2 i z R}\right|=\mathrm{e}^{\sigma-2 R y-2 \log (n \pi / R)}\left(1+\frac{\tau}{2 n \pi}\right)^{-\nu} \leq 1 / 6
$$

for sufficiently large $n$. Hence $\left|z^{-g} \varphi(z, 0)\right| \leq 25 / 72 \leq 5 / 12$ but $\left|z^{-g} \psi_{0}(z, 0)\right| \geq$ $11 / 12$ so that $\left|z^{-g} \psi(z, 0)\right| \geq 1 / 2$.

Second case: $|\operatorname{Im}(z)| \geq(\nu \log (n \pi / R)-\sigma+1) /(2 R)$ :

Here the main contribution comes from the term $c_{1} z^{-\nu} \mathrm{e}^{2 i z R}$. In fact, when $n$ is sufficiently large,

$$
\left|c_{1} z^{-\nu} \mathrm{e}^{2 i z R}\left(1+f_{1}(z)\right)\right| \geq \frac{11}{6} \geq \frac{7}{4}
$$

while $\left|z^{-g} \psi_{0}(z, 0)+f_{2}(z)\right| \leq 5 / 4$ so that $\left|z^{-g} \psi(z, 0)\right| \geq 1 / 2$.

Third case: $(\nu \log (n \pi / R)-\sigma-2) /(2 R) \leq|\operatorname{Im}(z)| \leq(\nu \log (n \pi / R)-\sigma+1) /(2 R)$ : We obtain firstly that

$$
\left|z^{-g} \psi(z, 0)\right| \geq\left|1+c_{1} z^{-\nu} \mathrm{e}^{2 i z R}\right|-\frac{1}{12}-\frac{3}{12}-\frac{1}{6} \geq \frac{1}{2}+\operatorname{Re}\left(c_{1} z^{-\nu} \mathrm{e}^{2 i z R}\right)
$$

since $\left|c_{1} z^{-\nu} \mathrm{e}^{2 i z R}\right| \leq 3$ when $n$ is sufficiently large.

Now let $\beta=\arg \left(c_{1} z^{-\nu} \mathrm{e}^{2 i z R}\right)=2 R x+\kappa-\nu \arg (z)$ and note that $\arg (z)=$ $3 \pi / 2 \pm \pi / 2+\arctan (y / x)$ where one has to choose the positive sign for positive $x$ and the negative sign for negative $x$ (recall that $y$ is negative in any case). After a small calculation one finds that $\pm 2 R x=2 n \pi+\tau+r(n)$ where $r(n)=O\left(\log (n)^{2} / n\right)$ as $n$ tends to infinity. This implies that $\arctan (y / x)=O(\log (n) / n)$ as $n$ tends to infinity. Hence

$$
\begin{aligned}
\cos (\beta) & =\cos \left(\kappa+\frac{3 \nu \pi}{2} \pm\left(\tau+\frac{\nu \pi}{2}+r(n)\right)-\nu \arctan (y / x)\right) \\
& \geq-|\sin ( \pm r(n)-\nu \arctan (y / x))| \geq-\frac{1}{18}
\end{aligned}
$$

provided that $\tau$ is chosen in such a way that $\cos (\kappa+3 \nu \pi / 2 \pm(\tau+\nu \pi / 2))$ is nonnegative for either choice of the sign. This can be achieved by choosing $\tau$ such that $\tau+\nu \pi / 2$ equals zero or $\pi$ depending on whether $\cos (\kappa+3 \nu \pi / 2)$ is nonnegative or not. Therefore we arrive at the following estimate

$$
\operatorname{Re}\left(c_{1} z^{-\nu} \mathrm{e}^{2 i z R}\right) \geq-3|\sin ( \pm r(n)-\nu \arctan (y / x))| \geq-\frac{1}{6}
$$

which holds for sufficiently large $n$.

Note that the estimates in the above proof were made to show that the circles can be chosen to avoid the resonances which are asymptotically located near the points where $1+c_{1} z^{-\nu} \mathrm{e}^{2 i z R}=0$. 
We will now show that the hypotheses of Lemma 5 can indeed be satisfied under the hypotheses we made earlier.

Lemma 6. Suppose $\left(q, q_{0}\right)$ satisfies Hypothesis 3. Then there is a number $\tau$ such that $|\psi(z, 0)| \geq|z|^{g} / 3$ for all $z$ on the semicircles given by $|z|=(2 n \pi+\tau) /(2 R)$ and $\operatorname{Im}(z) \leq 0$ and sufficiently large integers $n$.

Proof. We only have to prove that the hypothesis of Lemma 5 is satisfied. To this end define

$$
\gamma(t)=K(t, 0) \sum_{\ell=0}^{g} r_{\ell}(\xi(t)) z^{j-g}
$$

and consider the integral

$$
\int_{0}^{2 R} \gamma(t) \exp (i z t) d t
$$

According to Lemmas 2 and 3 this expression may be integrated by parts $n+1$ times. Thus we obtain

$$
\begin{aligned}
z^{-g} \varphi(z, 0) & =\left.\sum_{j=0}^{n+1}(-1)^{j} \gamma^{(j)}(t) \frac{\mathrm{e}^{i z t}}{(i z)^{j+1}}\right|_{0} ^{2 R}+(-1)^{n} \int_{0}^{2 R} \gamma^{(n+2)}(t) \frac{\mathrm{e}^{i z t}}{(i z)^{n+2}} d t \\
& =\sum_{j=0}^{n+1} \frac{\gamma^{(j)}(0)}{(-i z)^{j+1}}-\frac{\mathrm{e}^{2 i z R}}{(-i z)^{n+2}}\left(\gamma^{(n+1)}(2 R)-\int_{0}^{2 R} \gamma^{(n+2)}(t) \mathrm{e}^{i z(t-2 R)} d t\right)
\end{aligned}
$$

since $\gamma(2 R)=\ldots=\gamma^{(n)}(2 R)=0$. However,

$$
\gamma^{(n+1)}(2 R)=-\frac{1}{2^{n+2}}\left(q-q_{0}\right)^{(n)}(R) \sum_{\ell=0}^{g} r_{\ell}(\xi(2 R)) z^{j-g} \neq 0
$$

for all sufficiently large $z$.

The Riemann-Lebesgue lemma gives that $\int_{0}^{2 R} \gamma^{(n+2)}(t) \mathrm{e}^{i z(t-2 R)} d t$ tends to zero as $\operatorname{Re}(z)$ tends to infinity when $\operatorname{Im}(z)$ is fixed. A closer look at its proof reveals that this is in fact true uniformly in $\operatorname{Im}(z)$ as long as $\operatorname{Im}(z)$ is bounded above. Hence there is a positive $X$ such that

$$
\left|\int_{0}^{2 R} \gamma^{(n+2)}(t) \mathrm{e}^{i z(t-2 R)} d t\right| \leq \frac{\left|\gamma^{(n+1)}(2 R)\right|}{12}
$$

as long as $\operatorname{Im}(z) \leq 0$ and $|\operatorname{Re}(z)| \geq X$. It is also obvious that

$$
\left|\sum_{j=0}^{n+1} \frac{\gamma^{(j)}(0)}{(-i z)^{j+1}}\right| \leq \frac{1}{6}
$$

if $|z|$ is sufficiently large.

5.3. The zeros of the Jost function determine the potential. Suppose that $\left(q, q_{0}\right)$ satisfies Hypotheses 3 and let $\psi_{0}$ and $\psi$ be as before, in particular,

$$
\begin{gathered}
\psi(z, x)=\psi_{0}(z, x)+\int_{0}^{2 R} K(t, x) \psi_{0}(z, t) d t, \\
\psi^{\prime}(z, x)=\psi_{0}^{\prime}(z, x)+\int_{0}^{2 R} K^{(0,1)}(t, x) \psi_{0}(z, t) d t .
\end{gathered}
$$


and

$$
W(z)=\psi_{0}(z, x) \psi_{0}^{\prime}(-z, x)-\psi_{0}^{\prime}(z, x) \psi_{0}(-z, x) .
$$

Now define

$$
s(z, t, x)=\frac{1}{W(z)}\left(\psi_{0}(-z, x) \psi_{0}(z, t)-\psi_{0}(z, x) \psi_{0}(-z, t)\right)
$$

and $F(z, t, x)=-s(z, t, x)\left(q-q_{0}\right)(t)$. Then $\psi(z, \cdot)$ satisfies the integral equation $\psi(z, x)=\psi_{0}(z, x)+\int_{x}^{R} F(z, t, x) \psi(z, t) d t$. Therefore

$$
F^{\prime}(z, t, x)=M_{0}(z) F(z, t, x)-\frac{\psi_{0}(z, t)}{\psi_{0}(z, x)}\left(q-q_{0}\right)(t)
$$

and

$$
M(z)=M_{0}(z)-\frac{1}{\psi_{0}(z, 0) \psi(z, 0)} \int_{0}^{R} \psi_{0}(z, t) \psi(z, t)\left(q-q_{0}\right)(t) d t .
$$

Theorem 4. Suppose that $\left(q, q_{0}\right)$ satisfies Hypotheses 3 and that $q_{0} \in \mathcal{C}_{W}$ for some odd polynomial $W$. Then also $q \in \mathcal{C}_{W}$.

In particular, if none of the zeros of the Jost function $\psi(\cdot, 0)$ coincides with any of the zeros of $W$ then the zeros of $\psi(\cdot, 0)$ and their multiplicities determine $q$ uniquely.

Proof. Theorem 3 proves the existence of a function $\psi$ satisfying conditions (1) of Definition [1] Conditions (2) and (3) are satisfied because they hold for $\psi_{0}(z, \cdot)$. Condition (4) follows from equations (7) and (8). In particular, $\psi(\cdot, x)$ has growth order one since this is true for $\psi_{0}(\cdot, x)$ and since $K(\cdot, x)$ is compactly supported. Similarly, $\psi^{\prime}(\cdot, x)$ has growth order one. As $z$ tends to infinity along the imaginary axis $\psi_{0}(z, 0)$ approaches $z^{g}$ while, by Lemma $4 .(z, 0)$ tends to zero. This implies Condition (5).

We will now check condition (6) of the definition of the class $\mathcal{C}_{W}$. Suppose first that $\operatorname{Im}(z) \geq 0$. For sufficiently large $z$ we obtain from Lemma 4 that

$$
\frac{1}{2}|z|^{g} \leq|\psi(z, t)|,\left|\psi_{0}(z, t)\right| \leq \frac{3}{2}|z|^{g} .
$$

This and equation (91) gives

$$
\left|M(z)-M_{0}(z)\right| \leq 9\left\|q-q_{0}\right\|_{1}
$$

To estimate $M(z)$ for $z$ in the lower halfplane note that the Wronskian of $\psi(z, \cdot)$ and $\psi(-z, \cdot)$ satisfies

$$
W(\psi(z, \cdot), \psi(-z, \cdot))=W(z)
$$

Hence

$$
M(z)=M(-z)-\frac{W(z)}{\psi(z, 0) \psi(-z, 0)} .
$$

If $z$ is on the semicircles described in Lemma [ then, according to that Lemma, $|\psi(z, 0)| \geq|z|^{g} / 3$. This and the fact that $|W(z)| \leq C|z|^{2 g+1}$ imply that

$$
\left|M(z)-M_{0}(-z)\right| \leq\left|M(-z)-M_{0}(-z)\right|+\frac{|W(z)|}{|\psi(z, 0) \psi(-z, 0)|} \leq 9\left\|q-q_{0}\right\|_{1}+6 C|z| .
$$

The last statement of the theorem is now simply an application of Theorem 1 


\section{REFERENCES}

[1] Christer Bennewitz. A proof of the local Borg-Marchenko theorem. Comm. Math. Phys., 218(1):131-132, 2001.

[2] B. M. Brown, I. W. Knowles, and R. Weikard. On the inverse resonance problem. 2002, J. $L M S$, to appear.

[3] B. M. Brown, D. K. R. McCormack, W. D. Evans, and M. Plum. On the spectrum of secondorder differential operators with complex coefficients. R. Soc. Lond. Proc. Ser. A Math. Phys. Eng. Sci., 455(1984):1235-1257, 1999.

[4] B. M. Brown, R. A. Peacock, and R. Weikard. A local Borg-Marchenko theorem for complex potentials. J. Comput. Appl. Math., 148(1):115-131, 2002. On the occasion of the 65th birthday of Professor Michael Eastham.

[5] J. L. Burchnall and T. W. Chaundy. Commutative ordinary differential operators. Proc. London Math. Soc. Ser. 2, 21:420-440, 1923.

[6] J. L. Burchnall and T. W. Chaundy. Commutative ordinary differential operators. Proc. Roy. Soc. London, A 118:557-583, 1928.

[7] T. Christiansen. Resonances for steplike potentials: forward and inverse results. Preprint 2003.

[8] F. Gesztesy, K. Unterkofler, and R. Weikard. An explicit characterization of Calogero-Moser systems. Preprint: mp_arc (03-245), arxiv.org (nlin.SI/0305057), 2003.

[9] Fritz Gesztesy and Rudi Weikard. Picard potentials and Hill's equation on a torus. Acta Math., 176(1):73-107, 1996.

[10] Fritz Gesztesy and Rudi Weikard. Elliptic algebro-geometric solutions of the KdV and AKNS hierarchies - an analytic approach. Bull. Amer. Math. Soc. (N.S.), 35(4):271-317, 1998.

[11] A. R. Its and V. B. Matveev. Schrödinger operators with the finite-band spectrum and the $N$-soliton solutions of the Korteweg-de Vries equation. Teoret. Mat. Fiz., 23(1):51-68, 1975.

[12] Evgeni Korotyaev. Inverse resonance scattering on the half line. Preprint, 2000.

[13] Peter D. Lax. Integrals of nonlinear equations of evolution and solitary waves. Comm. Pure Appl. Math., 21:467-490, 1968.

[14] V. A. Marchenko. Reconstruction of the potential energy from the phases of scattered waves. Dokl. Akad. Nauk SSSR, 104(5):695-698, 1955. In Russian.

[15] Graeme Segal and George Wilson. Loop groups and equations of KdV type. Inst. Hautes Études Sci. Publ. Math., 61:5-65, 1985.

[16] Allen R. Sims. Secondary conditions for linear differential operators of the second order. J. Math. Mech., 6:247-285, 1957.

[17] R. Weikard. On rational and periodic solutions of stationary KdV equations. Doc. Math., 4:107-126 (electronic), 1999.

[18] R. Weikard. On commuting differential operators. Electron. J. Differential Equations, pages No. 19, 11 pp. (electronic), 2000.

[19] Maciej Zworski. A remark on isopolar potentials. SIAM J. Math. Anal., 32(6):1324-1326 (electronic), 2001.

Department of Computer Science, University of Wales, Cardiff, Po Box 916, Cardiff CF2 3XF, U.K.

E-mail address: Malcolm.Brown@cs.cf.ac.uk

Department of Mathematics, University of Alabama at Birmingham, Birmingham, AlABAMA 35294-1170, USA

E-mail address: rudi@math.uab.edu 